\title{
Spatial-Temporal Correlation-Based Low-Latency Compressed Sensing in WSNs
}

\author{
Jun Wang ${ }^{1,2}$, Shuqiang $\mathrm{Ji}^{1}$, Yong Cheng ${ }^{2}$ \\ ${ }^{1}$ Dept. of Computer \& Software, Nanjing University of Information Science \& \\ Technology, Nanjing China \\ ${ }^{2}$ Dept. of Information Construction \& Management, Nanjing University of \\ Information Science \& Technology, Nanjing 210044, China \\ shuqiangji@foxmail.com
}

\begin{abstract}
Wireless Sensor Networks (WSNs) have characteristics of large size, limited resources, large amount of transmission data, and so on. In order to reduce the redundancy of sensed data and decrease network data traffic. We applied CS to clustered structure, proposed Low-Latency Compressed Sensing model (LLCS) which is based on the spatialtemporal correlation of sensed data, the model is also capable of processing sparse abnormal events which is a crucial feature in WSNs. We analyzed the relationship between compression ratio and sampling rounds and verified the abnormal event processing method. The results of simulation experiments using the real data show that LLCS could reduce data transfer volume significantly and process abnormal readings effectively.
\end{abstract}

Keywords: wireless sensor networks; compressed sensing; spatial-temporal correlation; low latency

\section{Introduction}

Wireless Sensor Networks (WSNs) [1] are comprised of a large number of sensor nodes, which is consist of sensing, data processing, and communicating components. Sensor nodes cooperatively sense, collect and process information of interesting phenomenon. Eventually, they send the processed information to sink. WSNs have become a hot research field because of its extensive application prospect.

The purpose of WSNs is to gather raw data collected by sensor nodes with small size. Sensor nodes are densely deployed either inside or very close to the interesting phenomenon. The amount of transmission data will be very large if sending all raw data to sink, then it will lead to network congestion and shorten the life of WSNs because Sensor nodes have highly constrained energy resources. The computation and storage operations of nodes require less energy than transmission, decreasing the amount of transmission data will reduce the energy consumption significantly and prolong the lifetime of WSNs. Thus, it's necessary to save energy and prolong the lifetime of the network through decreasing transmission data.

Compressed sensing (CS) [2-4] has opened a new avenue of data acquiring in WSNs. If a signal is sparse or sparse in a sparse basis, then we can project the signal onto a measurement matrix which is uncorrelated to the sparse basis and get a few measurements. And finally, reconstruct the original signal with high probability by solving the convex optimization problem.

WSNs are one of the most important fields in which CS can be employed, compared with many other data compression technology CS has many advantages. Firstly, the work of encoder is very simple; each sensor obtains measurements by projecting the signal onto 
a measurement matrix. Secondly, CS provides effective data compression, which can reconstruct the $K$ sparse signal in $R^{N}$ from $M \approx 4 K$ measurements. Thirdly, each sensor encodes its measurements independently, and different decoder scheme [5-6] can be used to reconstruct the original signal from the same random measurements. In addition, robustness is one of the properties of CS; it's possible to reconstruct the signal even though a few data was missing in the process of transmission. If the signal gathered by a sensor node has sparse representation in one basis, then low complexity coding algorithm can be applied to obtain random measurements in the sensor node with constrained computing and storage capacity. The original signal can be recovered from measurements by running the high complexity decoding algorithm in the sink with powerful computational capacity and enough energy.

Spatial correlation usually exists among the readings of close nodes because of redundant deployment. A lot of work had been done to compress signals based on spatial correlation. Temporal correlation usually exits among the readings of nearby time instant in the same sensor node, such spatial-temporal correlation encourages us to improve the efficiency of data acquisition for WSNs by incorporating CS. Many methods and model based on the spatio-temporal correlation result in higher latency, so it's also necessary to research the problem about reducing the latency. Since the clustering method has many advantages over other methods [21-23], such as fault tolerance and traffic load balancing, we applied CS to clustered structure in sensor networks. In this paper, we proposed LowLatency Compressed Sensing model, compressing and reconstructing the sensed data by making full use of the spatial-temporal correlation during an interval. Sparse abnormal event processing is also a crucial feature in WSNs. In this paper, sparse abnormal event processing method is also proposed to trigger notification of the potential danger.

The rest of the paper was organized as follows: Section 2 describes the existing methods for data compression with $\mathrm{CS}$ and discuss about their advantages and disadvantages. Section 3 describes spatial-temporal correlation-based Low-Latency Compressed Sensing model in details. Section 4 simulates the model by using real experimental data. The last section concludes this paper.

\section{Related Works}

A comprehensive comparison of data compression techniques for WSNs can be found in [12]. CWS [13] is a special implementation of CS for distributed spatial sampling in WSNs. CWS models a WSN as a distributed sampling system in which every single sensor node record the local value, get measurements by using distributed method for computing the matrix multiplication, and then reconstruct the signal in the sink. CDG [14] is one of the first detailed implementation of CWS for large-scale WSNs. The paper shows that CS leads to a more efficient and stable signal acquisition technique compared to some other traditional methods. CDG is also able to tackle abnormal sensor readings and detect events. CDG makes use of spatial correlation, but do not take temporal correlation into account. DCS [7-9] is an extension of the pure spatial CS-based data acquisition to the temporal domain. DCS introduces three different joint sparsity models that apply in different situations. It equips each sensor node with its own independent random measurement matrix, and, reconstructs the signal using a joint recovery algorithm. DCS exploits not only spatial but also temporal compressibility. DCS requires much less measurements than applying CS to single node when the number of sensor nodes is very large. In the study [15] researchers propose a clustering method that used hybrid CS for sensor networks and aim at finding the optimal size of clusters that can lead to minimum number of transmissions. However, there is no detail discussion about an application model based on CS in this paper. Haifeng Hu presents Spatial Correlationbased Distributed Compressed Sensing (SCDCS) model and algorithm in [16]. After calculating the radius of event area and forming a cluster, the cluster head performs the 
SCDCS encoding algorithm to get measurements. Then the SCDCS decoding algorithm is performed at the sink node to joint recover the sensed data. SCDCS also do not take temporal correlation into account. The researchers develop a novel CS-based concept of sampling window as an efficient spatio-temporal signal acquisition technique targeting the dominant WSN deployments of multi-hop WSNs [17]. The model guarantees balanced energy consumption by the sensor nodes in a multi-hop topology to prevent overloaded nodes and network partitioning, and consider spatial and temporal correlations. It is also capable of abnormal event detection. However, the model will lead to high transmission delay when the scale of the network is too large.

Low-Latency Compressed Sensing model compresses the signal with taking spatialtemporal correlation into consideration and obtain low-latency data transmission with appropriate measurement matrix. In addition, our sparse abnormal event processing method is capable of sending abnormal readings to sink quickly and reducing the effects of abnormal readings on data compression in cluster heads.

\section{Low Latency Compressed Sensing Model}

\subsection{Compressed Sensing}

The theory of Compressed Sensing shows that CS will compress the signal adequately while acquiring it. CS combines data acquisition and compression. Compressed sensing is comprised of sparse representation, random measurement and reconstruction.

There is a signal $x$ which can be viewed as a column vector in $R^{N}$ with elements $x_{1} x_{2} \ldots x_{N}$. The signal is $K$ sparse if only $K$ elements in $x$ are nonzero and other $N-K$ elements are zero or can be ignored compared with the $K$ elements. That is $\|x\|_{0}=K(K \square N)$ however, natural signals $f\left(f \in R^{N}\right)$ are almost not sparse. Any signal in $R^{N}$ can be represented in terms of a basis of $\mathrm{N} \times 1$ vectors $\left\{\psi_{i}\right\}_{i=1}^{N}$. For simplicity, assume that the basis is orthonormal. Then, the signal $f$ can be expressed as:

$$
f=\sum_{i=1}^{N} \theta_{i} \psi_{i}
$$

or $f=\psi \theta, \psi$ is an $N \times N$ matrix. $\theta$ is the column vector of weighting coefficients $\theta_{i}$. If most of the elements in $\theta$ are zero or near zero, $\theta$ is sparse and call $\psi$ the sparse basis. Clearly, $f$ and $\theta$ are equivalent representations of the signal, with $f$ in the time or spatial domain and $\theta$ in the $\psi$ domain. Most signals recorded from natural phenomena are compressible under the Fourier transform, Discrete Cosine Transform (DCT) and Discrete Wave Transform (DWT) [18]. This is the fundamental of compression of natural signals.

The natural signal $f$ is $K$ sparse in $\psi$ domain, then it is possible to construct an $M \times N(M \square N)$ measurement matrix $\phi$ which is irrelevant to the sparse basis $\psi$. We could acquire $M$ linear random measurements by projecting $f$ onto the measurement matrix $\phi$. Assume $y \in R^{M}$ represents these measurements such that $y=\phi f$. Because of $f=\psi \theta, y$ can also be represented as $y=\phi f=\phi \psi \theta=A \theta(A=\phi \psi)$ and $A$ was called the sensing matrix. The sensing matrix $A$ needs to obey Restricted Isometry Property (RIP) [19]. Certain matrices such as Gaussian random measurement matrix [6], Fourier random measurement matrix could be used as the measurement matrix.

It is possible to recover $f$ from $y$ by solving the convex optimization problem:

$$
\bar{\theta}=\arg \min \|\theta\|_{1} \theta \in R^{N} \text { subject to } y=\phi \psi \theta
$$


Then, natural signal could be recovered by inverse transformation $\bar{f}=\psi \bar{\theta}$.

Signal recovery is high possible when the number of measurements follows

$$
M>c K \log { }^{N / K}
$$

Where $c>1$ is a small real constant.

\subsection{Model Building}

Spatial-Temporal correlation usually exists among the readings of close nodes during an interval. So, we proposed Low-latency Compressed Sensing model based on the spatial-temporal correlation of sensed data.

We firstly assume that sensor nodes in WSNs are all synchronized, sensor nodes do not need to transmit sensed data to sink immediately. There are $n$ sensor nodes in a cluster, these sensor nodes gather data and send it to the cluster head. Then, the cluster head process the data set and transmit the processed data to sink by multiple hops routing, finally, the data set would be reconstructed in the sink.

Clusters would be built up after running the clustering algorithm in the WSNs according to the study [15]. Then, nodes in the cluster start to gather data. The cluster head can build up a vector $f\left(f=\left[\begin{array}{llll}x_{1} & x_{2} & \ldots & x_{n}\end{array}\right]\right)$ of size $n$ after receiving the readings collected by member nodes at the same time instant. Spatial correlation exits in $f$, and $f$ is sparse under DCT or DWT. The relationship between compression ratio and clusters of different size is shown in figure 1 when compress the signal based on spatial correlation with data set [24].

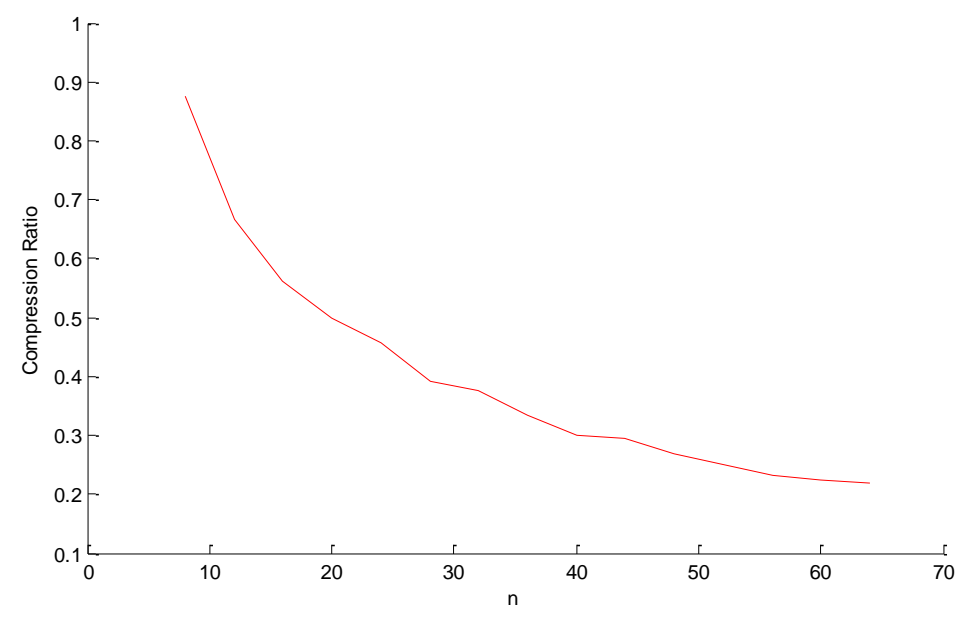

Figure 1. The Relationship between the Node Number and Compression Ratio with the Same Error of Reconstruction

Figure 1 shows that it is meaningless to compress the signal based on spatial correlation when there are only a small number of nodes in the cluster. The number of required compressive measurements, namely the parameter $M$ in Equation (3), grows logarithmically with the dimension of the signal under the condition that the sparsity of the signal is stable. So, the desirable logarithmic cost can be exploited by increasing the temporal sampling rate of individual sensor nodes.

During the interval $\left[t_{0}, t_{T}\right]$, sensor nodes in a cluster sample $T$ times and $T$ called sampling rounds. At time instant $t$, putting samples from each member node together, a discrete spatial signal is composed that can be represented by a 
vector $f_{t}\left(f_{t} \in R^{n} f_{t}=\left[\begin{array}{llll}x_{1} & x_{2} & \ldots & x_{n}\end{array}\right]\right)$. After $T$ sampling rounds, the vector can be represented as $f=\left[f_{1}^{\prime} f_{2}^{\prime} \ldots f_{T}^{\prime}\right]\left(f \in R^{N} N=n \times T\right), f$ is sparse under specific transform basis that is $f=\psi \theta\left(\|\theta\|_{0}=K\right.$ ). Then, Gaussian random measurement matrix $\phi$ ( $\phi$ is an $M \times N(M<<N)$ real matrix) can be calculated by using a pseudorandom number generator which is common between sensor node and the sink. When the Gaussian random number generator at every cluster head is initialized by the id-number of that cluster head, the sink can exactly reproduce the measurement matrix. The measurement matrix does not need to be stored on the cluster head in this case. The measurement vector $y \quad\left(y \in R^{M}\right)$ can be got by projecting ${ }^{f}$ onto the measurement matrix $\phi$ that is $y=\phi f$. After that, the cluster head transmits the measurement vector $y$, id-number, timestamp $t_{0}$ to the sink by multi-hop routing. In the end, the original signal can be reconstructed with reproduced measurement matrix in the sink. The LLCS model is shown as Figure 2.

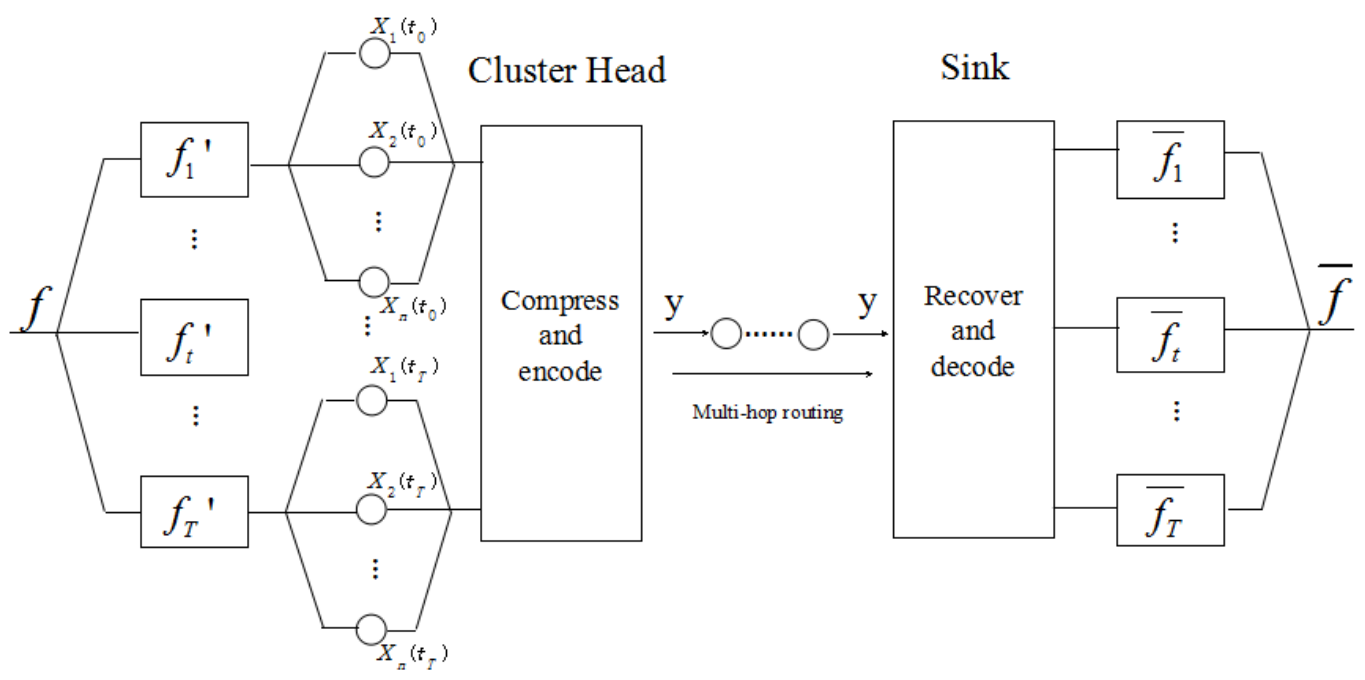

Figure 2. Low-Latency Compressed Sensing model

\subsection{Compression and Reconstruction}

Yap et al. [10] have shown that block-diagonal random measurement matrices can perform as good as dense random measurement matrix in CS signal acquisition and recovery. So we also use block-diagonal measurement matrix. Firstly, the cluster head generates $T$ seeds with pre-installed pseudo random number generator using its own idnumber. Then, generate $T \quad m \times n$ submatrices $f=\psi \theta\left(\|\theta\|_{0}=K\right)$ using the $T$ seeds in the cluster head. The measurement matrix could be composed with these submatrices.

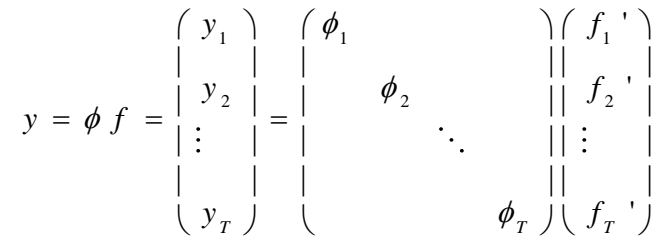

In Equation (4) each block corresponds to samplings at a time instant. Let $\phi_{t}$ and $f_{t}$ denote the measurement matrix and the spatial signal at time $t$ respectively. The measurement vector will be $y_{t}=\phi_{t} f_{t}$ at time $t$. Then transmit a data packet with $y_{t}$, the 
seed generating $\phi_{t}$, and timestamp $t$ to the sink. The cluster head needs to send the data packet $T$ times in an interval. After receiving the data packet, a new measurement vector could be composed with $y_{t}$ and previous $T-1$ data packets. That is Equation (5).

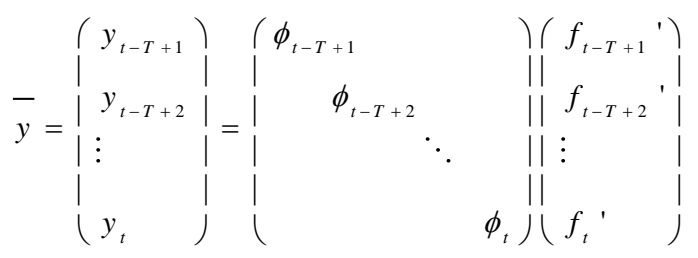

The measurement vector in interval $[t-T+1, t]$ is represented as $\bar{y}$. Only the last set of data needs to be kept after recovering the signal. In the sink, recovery algorithm starts to recover the signal after rebuilding the measurement vector $\bar{y}$. The decoding algorithms commonly used to solve a convex optimization problem that conveniently reduces to a linear program are Basic Pursuit (BP) [5], Orthogonal Matching Pursuit (OMP) [6] and so on. Then, the closely approximate compressible signal $\bar{f}$ will be got by the inverse transformation $\bar{f}=\psi \bar{\theta}$.

The model is less dependent on the storage capacity of cluster heads and it shows that the delay affects measurements acquisition and signal recovery only at initialization. Delay refers to the time required to acquire measurements from the network and not the time required by the recovery algorithm to reconstruct the spatial-temporal signal. The recovery algorithm starts to recover the signal after receiving original $T$ data packets. Then, acquiring measurements and reconstructing the signal can be done seamlessly. After rotating cluster head, the new measurement matrix and new measurement vector can also be reconstructed in the sink when sink received data packets from the same cluster.

\subsection{Recover Signal with Sparse Abnormal Readings}

Abnormal event detection and processing is a crucial feature in WSNs, So, sending the abnormal data to sink quickly and triggering notification about potentially harmful situations are important tasks of WSNs.

When abnormal events take place, the sparsity of sensor readings is compromised. As an example, Figure 3 differs from figure 4 only by two abnormal readings. The corresponding DCT coefficients shown in figure 4 are not sparse any more. Therefore, the signal in figure 4 is not sparse in either time domain or transform domain. In this situation, we cannot compress and recover the signal effectively. Over-complete dictionaries had been proposed to detect and handle sparse abnormal sensor readings in [14-17]. In this paper, we proposed a new method to process abnormal readings.

At time $t$, sensed data $f_{t}$ contains an abnormal reading $x_{u}$. Under normal conditions, adjacent readings in sensed data are similar because of spatial correlation. So, a new vector $f_{t}\left(f_{t}=\left[\begin{array}{ll}x_{1} \ldots x_{u-1} & x_{u-1} \ldots x_{n}\end{array}\right]\right)$ can be reconstructed by replacing $x_{u}$ with $x_{u-1}$. If the first reading $x_{1}$ of $f_{t}$ is abnormal, replace it with $x_{2}$, then pack abnormal reading $x_{u}$, timestamp $t$, location $u$ and transmit to the sink. The original signal will be changed to $f=\left[f_{1}{ }^{\prime} \ldots f_{t}{ }^{\prime} \ldots f_{T}{ }^{\prime}\right]\left(f \in R^{N} N=n \times T\right)$. Then, compress the signal and transmit the measurement vector to the sink. 

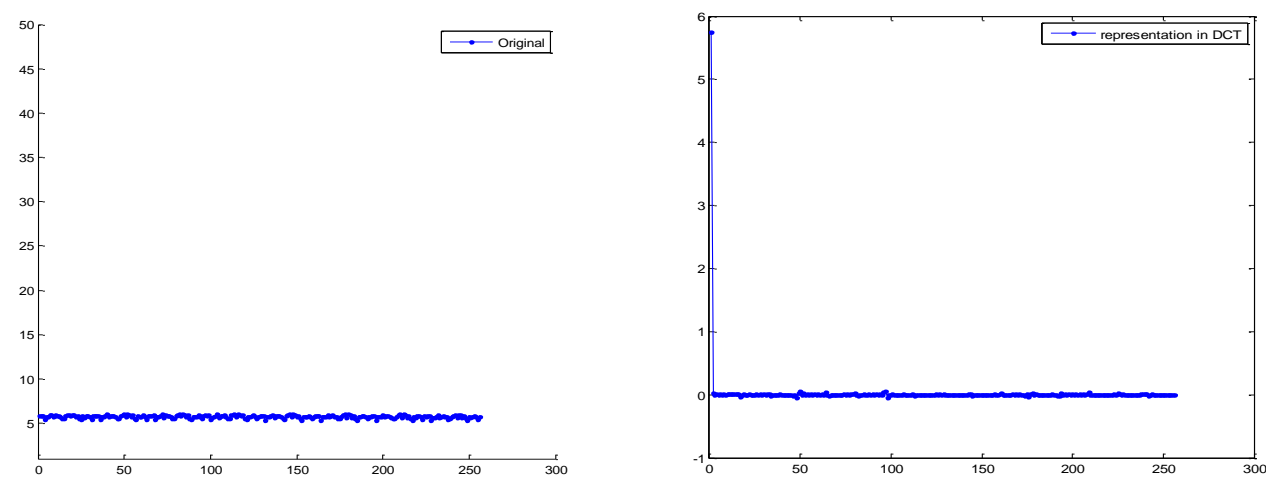

Figure 3. A Normal Signal in DCT Domain
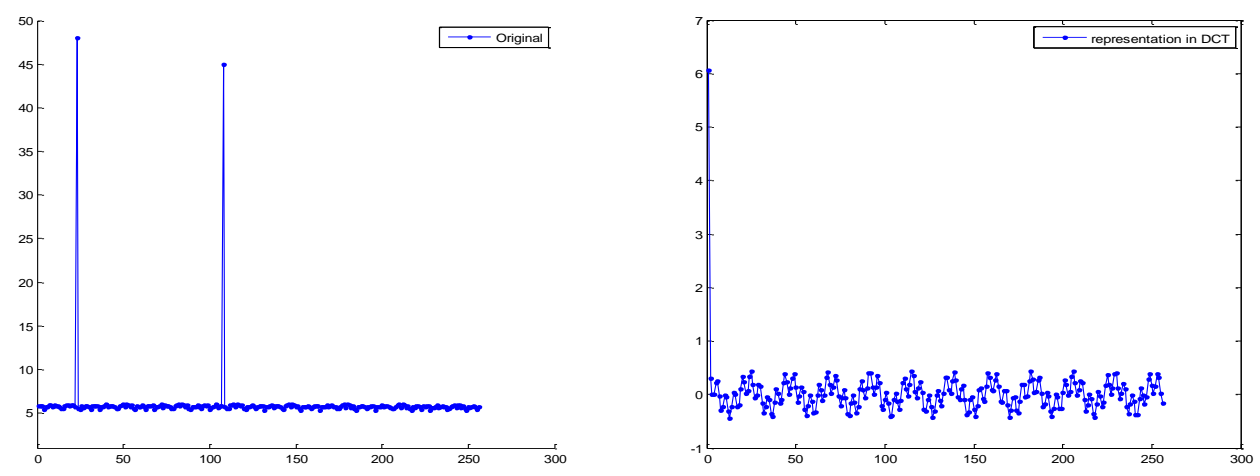

Figure 4. A Signal with Two Abnormal Readings in Dct Domain

The notification about potentially harmful situation will be triggered when the sink received the abnormal data packet. The closely approximate compressible signal $\bar{f}$ could be got by replacing corresponding data according to the timestamp and location of abnormal readings.

\section{Performance Evaluation}

In simulation, we discuss in terms of the relationship between the reconstruction error and sampling rounds, the relationship between compression ratio and sampling rounds when achieving the same reconstruction error. we compare the performance results of compression based on spatial-temporal correlation with only spatial correlation-based. In addition, the sparse abnormal event processing method is also verified.

The LLCS model is evaluated with real-world data collected by the LUCE WSN deployment at EPFL [24] by using Matlab, the measurement matrix use Gaussian random measurement matrix and recovery algorithm adopt OMP [6]. The ambient temperature values of 8 sensor nodes and 32 sensor nodes corresponding to the quantity of nodes in a cluster are used as the physical parameter for evaluating the model. The sampling interval is 1 minute. In the LUCE dataset, some readings were too desynchronized. Therefore, we have preprocessed the dataset while preserving the attributes of the spatial-temporal signal to have a synchronized data set. The accuracy of the signal reconstruction is measured by the Relative Error $\varepsilon$. In order to avoid extreme situations we adopt Mean Relative Error by running recovery algorithm 400 times. 


$$
\varepsilon=\frac{\|\bar{f}-f\|_{2}}{\|f\|_{2}}
$$

Figure 1 shows that it is meaningless to compress the signal based on spatial correlation when there are only a small number of nodes in the cluster. So, we compress signals by extending signals to temporal domain.

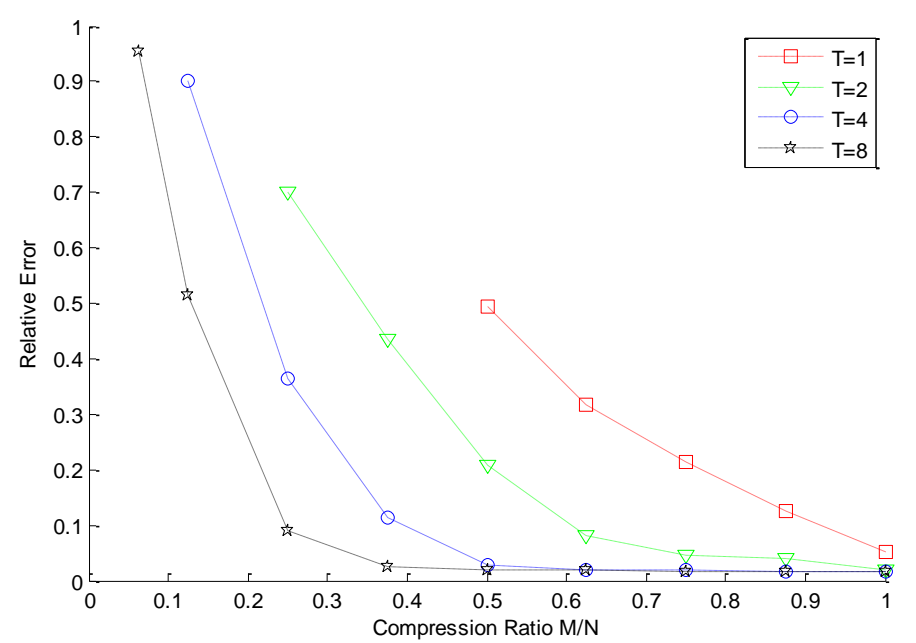

Figure 5. The Relative Error for Different Compression Ratio When Only 8 Nodes in A Cluster

The compression ratio $(\mathrm{CR})$ is defined as the ratio of the number of measurements to the number of the sensed data. The evaluations are first done for $T=1$. However, figure 5 shows that the original signal cannot be recovered when compression ratio is less than 0.5 when there are 8 sensor nodes in a cluster. Then, the evaluation is repeated for $T=2$ $T=4$ and $T=8$. For larger $T$, lower relative error or higher reconstruction accuracy is possible for lower $M / N$. And the reconstruction error tends to be zero when the number of measurements is approaching the number of the sensed data. Reconstruction error is 0.45 when $T=1$ and $C R=0.5$. In contrast, reconstruction error is only 0.019 when $T=8$ with the same compression ratio as shown in figure 5 . Therefore, the model is suitable for the cluster with a small number of sensor nodes.

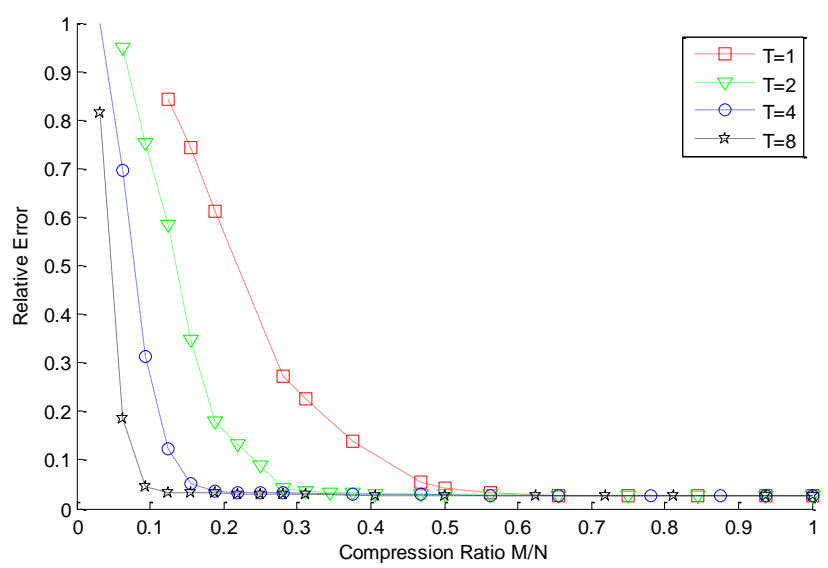

Figure 6. The Relative Error in the Number of Measurements when there are 32 Sensor Nodes in a Cluster 
Lower relative error could be achieved by making use of spatial correlation when there are much more sensor nodes in a cluster such as 32 sensor nodes. As shown in figure 6 , if the relative error is expected to be less than 0.032 , compression ratio needs to be greater than 0.56 when $T=1$ which is basically equivalent to the plain spatial sampling case. That is, 144 measurements need to be used to recover the original signal which is consist of 256 readings when $T=8$. In contrast, only about 48 measurements or $C R=0.187$ need to be used to achieve the same relative error when the original signal is compressed based on spatial-temporal correlation. Figure 6 also shows the relative error is approximately inversely proportional to the number of measurements. So, LLCS model reduce data transmission significantly, decrease energy consumption of nodes and prolong the lifetime of WSNs effectively.

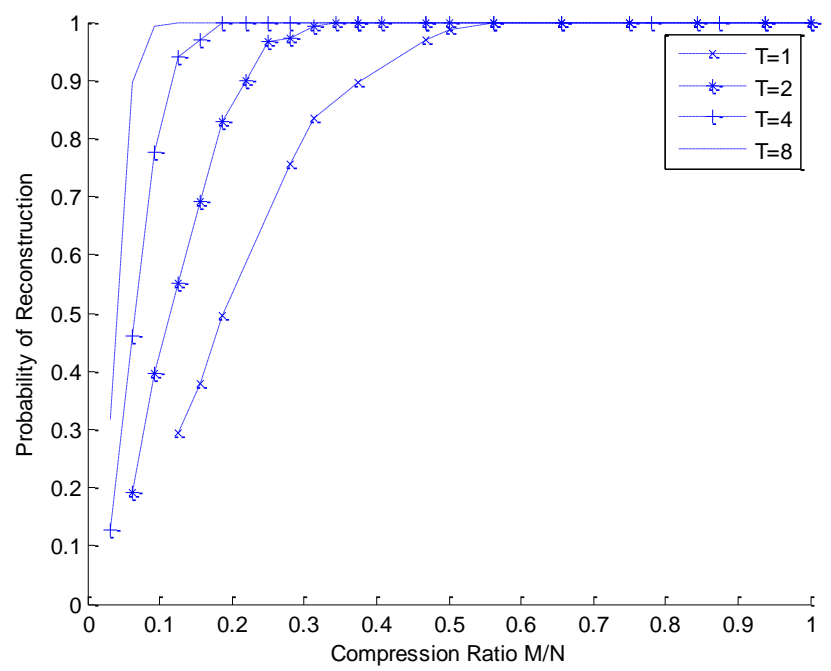

Figure 7. The Probability of Reconstruction for Different Sampling Rounds

We make the relative error less than 0.05 as the criterion of successful recovery.

Figure 7 shows that, the probability of recovering signal successfully is proportional to the number of measurements. And for larger $T$, higher probability is possible for lower $M / N$ or less measurements. Therefore, compressing the original signal based on spatial-temporal correlation, not only decrease the transmission data significantly, but also increase the probability of successful reconstruction.

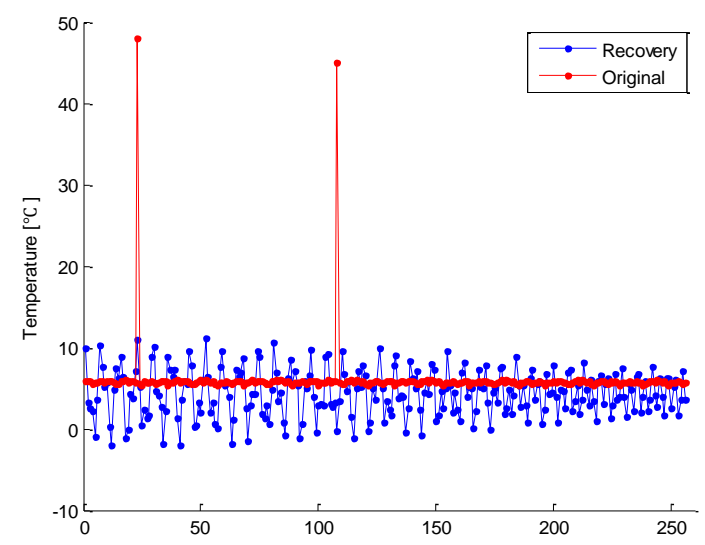

Figure 8. Signal Reconstruction without Processing 
Rare abnormal readings are simulated by deliberately modifying the two readings in the original signal when $T=8$. We increased these two values at random. This may resemble a fire starting in the forest. We compress and recover the signal with no preprocessing firstly and then process it with our method. Measurements are set as $M=48$ and sparsity $K=2$.

As shown in Figure 8, the effect of recovery is very poor, and the relative error even reach 0.61 . Moreover, the two abnormal readings cannot be recovered accurately. Then, the same signal is processed with our method.

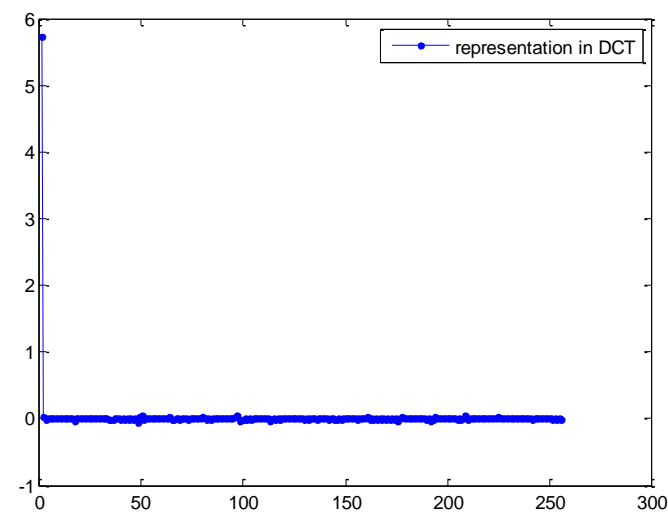

Figure 9. Representation in Dct after Processing

Figure 9 shows that after processing, the original signal can be represented in DCT, that is, it satisfies the premise of CS.

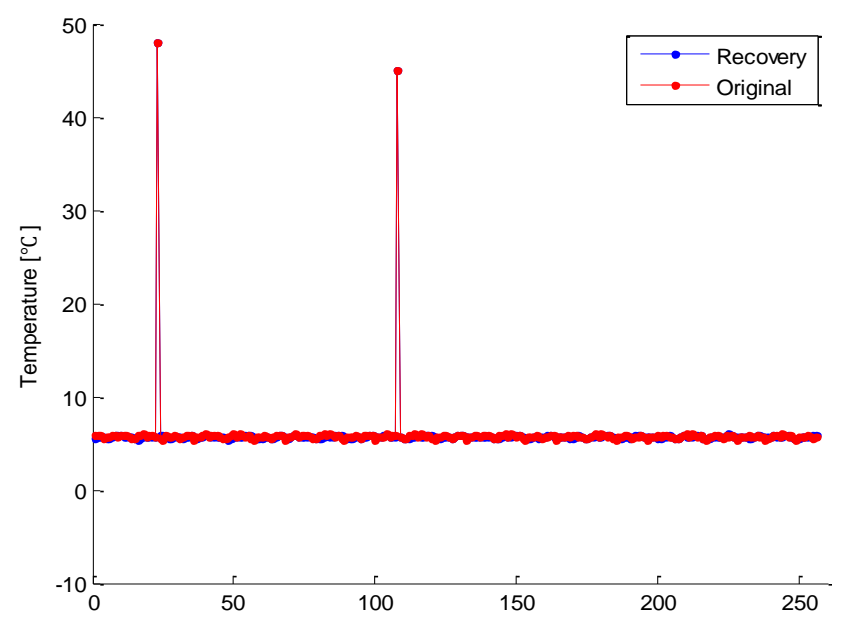

\section{Figure 10. Signal Reconstruction after Processing}

The relative error is only 0.03 after the original signal being processed with our method as shown in figure 10. In particular, the cluster head could transmit abnormal readings immediately after receiving readings at time $t$, and the delay would be only the transmission time. Moreover, we decrease the impact of abnormal readings on data compression in cluster heads. 


\section{Conclusions and Further Work}

In this paper, we applied CS to clusters, proposed Low-Latency Compressed Sensing model based on spatial-temporal correlation and discussed sparse abnormal events processing method. Then, we analyzed in terms of the relationship between the reconstruction error and sampling rounds, the probability of reconstruction and sampling rounds. The results of simulation show that LLCS model is suitable for clusters of different scale, and LLCS can obtain better compression performance. The model reduced the communication traffic, prolonged the life of the network significantly and decreased delay, moreover, LLCS is also capable of processing sparse abnormal events. In the future, we will further seek to improve the efficiency of compression and reconstruction, and decrease transmission delay.

\section{Acknowledgments}

The work in this paper has been supported by the National Natural Science Foundation of China (Grant No. 61373064, 61402236); Open Fund for Jiangsu Key Laboratory of Agricultural Meteorology(Grant No.KYQ1309); Six talent peaks project in Jiangsu Province(Grant No.2013-DZXX-019);Jiangsu Province Research Prospective Joint Research Project(Grant No.BY2014007-2);R\&D Special Fund for Public Welfare Industry(Grant No.GYHY201106037).

\section{References}

[1] I. F. Akyildiz, W. Su, Y. Sankarasubramaniam, "Wireless sensor networks:a survey", Computer Networks, vol. 38, (2002), pp. 393-422.

[2] D. L. Donoho, "Compressed sensing", IEEE Transaction on Information Theory, vol. 52, no. 4, (2006), pp. 1289-1306.

[3] R. G. Baraniuk, "Compressive sensing”, IEEE Signal Processing Magazine, vol. 24, no. 4, (2007), pp.118-124.

[4] E. J. Candès and M. B. Wakin, "An Introduction to compressive sampling", IEEE Signal Processing Magazine, vol. 25, no. 2, (2008), pp. 21-30.

[5] E. J. Candès and T. Tao, "Near-optimal signal recovery from random projections", Universal encoding strategies, IEEE Transaction Information Theory, vol. 52, no. 12, (2006), pp.5406-5425.

[6] J. A. Tropp, A. C. Gilbert, "Signal recovery from random measurements via orthogonal matching pursuit" IEEE Transaction on Information Theory, vol. 53, no. 12, (2007), pp. 4655-4666.

[7] D. Baron, M. B. Wakin, M. F. Duarte, S and Sarvotham, "Distributed compressed sensing", (2005), Available at dsp.rice.edu/cs.

[8] M. F. Duarte, S. Sarvotham and D. Baron, "Distributed compressed sensing of jointly sparse", in Conference Record of the Thirty-Ninth Asilomar Conference on Signals, Signals, Systems and Computers, (2005), pp.1537-1541.

[9] M. F. Duarte, M. B. Warkin and D. Baron, "Universal distributed sensing via random projections", in Proc. of the $5^{\text {th }}$ international conference on Information processing in sensor networks (IPSN), (2006), pp.177-185.

[10] H. L. Yap, E. Armin and M. B. Warkin, "The restricted isometry property for block diagonal matrices", in Proc. of $45^{\text {th }}$ Annual Conference on Information Sciences and Systems(CISS), Baltimore, MD, (2011), pp.1-6.

[11] E. J. Candès, J. K. Romberg and T. Tao, "Stable signal recovery from incomplete and inaccurate measurements", Communications on Pure and Applied Mathematics, vol. 59, no. 8, (2006), pp.12071223.

[12] T. Srisooksai, K. Keamarungsi, P. Lamsrichan and K. Araki, "Practical data compression in wireless sensor networks", a survey Journal of Network and Computer Applications, vol. 35, no. 1, (2012), pp. 37 -59 .

[13] W. Bajwa et al., "Compressive wireless sensing", in Proc. of the $5^{\text {th }}$ international conference on Information processing in sensor networks(IPSN), Nashville, TN, (2006), pp. 134-142.

[14] C. Luo, F. Wu and J. Sun, "Compressive data gathering for large-scale wireless sensor networks", in Proc. of the 15th annual international conference on Mobile computing and networking (Mobicom), Beijing, China, (2009) pp. 145-156.

[15] R. Xie and X Jia, "Transmission efficient clustering method for wireless sensor networks using compressive sensing", IEEE Transactions on Parallel and Distributed Systems, vol. 25, no. 3, (2013), pp. 806-815. 
[16] H. Hu and Z. Yang, "Spatial correlation-based distributed compressed sensing in wireless sensor networks", in Proc. Of $6^{\text {th }}$ International Conference on wireless communications networking and mobile computing (WICOM), Chengdu, (2010), pp.1-4.

[17] M. Mahmudimanesh, A. Khelil and N. Suri, "Balanced spatio-temporal compressive sensing for multihop wireless sensor network", in Proc. of $9^{\text {th }}$ International conference on Mobile Adhoc and Sensor Systems(MASS), Las Vegas, NV, (2012) pp.389-397.

[18] A. Graps, "An introduction to wavelets", IEEE Computer Science Engineering, vol. 2, no. 2, (1995) pp. 50-61.

[19] E. J. Candès, J. Romberg and T. Tao, "Robust uncertainty principles: Exact signal reconstruction from highly incomplete frequency information", IEEE Transactions on Information Theory, vol. 52, no. 2, (2006), pp.489-509.

[20] C. T. Chou, R. Rana and W. Hu, "Energy efficient information collection in wireless sensor networks uses adaptive compressive sensing", in Proc. of IEE $34^{\text {th }}$ conference on local computer networks, Zurich, (2009), pp.443-450.

[21] M. Youssef, A. Youssef, and M.Younis, "Overlapping multihop clustering for wireless sensor networks", IEEE Trans, Parallel Distrib, Syst., vol. 20, no. 5, (2009), pp.955-972.

[22] S. Soro and W. B. Heinzelman, "Cluster head election techniques for coverage preservation in wireless sensor networks", Ad Hoc Networks, vol. 7, no. 5, (2009), pp.955-972.

[23] W. Heinzelman, A. Chandrakasan and H. Balakrishnan, "An application-specific protocol architecture for wireless microsensor networks", IEEE Trans. Wireless Commun., vol. 1, no. 4, (2002), pp.660-670.

[24] Sensorscope LUCE deployment on the EPFL campus (2008), Available,

[25] http://lcav.epfl.ch/cms/lang/en/pid/86035 